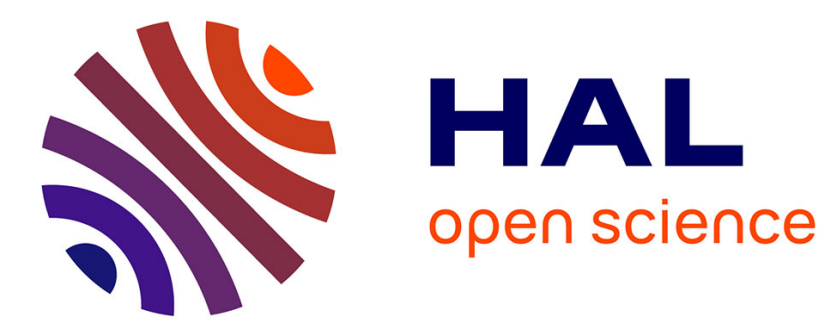

\title{
CATHODOLUMINESCENCE QUANTUM WELL STUDIES
}

\author{
C. Warwick
}

\section{To cite this version:}

C. Warwick. CATHODOLUMINESCENCE QUANTUM WELL STUDIES. Journal de Physique IV Proceedings, 1991, 01 (C6), pp.C6-117-C6-123. 10.1051/jp4:1991619 . jpa-00250704

\section{HAL Id: jpa-00250704 https://hal.science/jpa-00250704}

Submitted on 1 Jan 1991

HAL is a multi-disciplinary open access archive for the deposit and dissemination of scientific research documents, whether they are published or not. The documents may come from teaching and research institutions in France or abroad, or from public or private research centers.
L'archive ouverte pluridisciplinaire HAL, est destinée au dépôt et à la diffusion de documents scientifiques de niveau recherche, publiés ou non, émanant des établissements d'enseignement et de recherche français ou étrangers, des laboratoires publics ou privés. 


\title{
CATHODOLUMINESCENCE QUANTUM WELL STUDIES
}

\author{
C.A. WARWICK \\ AT \& T Bell Laboratories, Room 4C-410, Crawfords Corner Road, Holmdel, NJ 07733- \\ 1988, USA
}

\begin{abstract}
The advent of quantum well structures has given rise to new physics and new technology. Quantum well propertiees are determined by interfaces between chemically distinct, heterostructural compounds. The study of these interfaces is of vital importance to the progress of this field, and many studies using a variety of techniques have been made. The scanning cathodoluminescence microscope has made major contributions to the understanding of interfaces in quantum well devices but has previously been limited to a spatial resolution of $\approx 1 \mu \mathrm{m}$. We have achieved $60 \mathrm{~nm}$ spatial resolution in this mode by: 1) using a small probe generated by an intense field emission source; 2) using a $1 \approx 2 \mathrm{kV}$ beam to inject both near the surface and with minimal lateral scattering $(10 \approx 30 \mathrm{~nm})$; and 3$)$ by detecting photons from pre-diffusion radiative recombination events. Carriers living longer than $\approx 10 \mathrm{ps}$ recombine non-radiatively at the surface; the surface acts as a picosecond "shutter". We force surface recombination to dominate the lifetime by injecting very close to the surface.
\end{abstract}

\section{1.-Introduction}

The ease of manufacture of silicon solid state electronics has fuelled the information age. However, in some applications, specifically those involving the transmission of data by guided electromagnetic waves, electronics has a severe handicap. Electronic devices can presently generate electromagnetic waves up to frequencies of many $\mathrm{GHz}$, but these waves suffer strong absorption in their guiding media (eg copper wave-guides, co-axial cable). The metallic conduction band electrons in the guiding media respond to the wave, and undergo a dissipative motion. As a result the signal is lost to heat. In contrast, for waves at much higher frequencies, in the $\approx 200 \mathrm{THz}$ (infra-red) range, the interaction with the atomic shell electrons in certain glasses is almost lossless. Indeed, with high purity silica fibers, and appropriate selection of photon energy, $(e g 1.55 \mu \mathrm{m})$, losses can be reduced to as low as $0.154 \mathrm{~dB} \mathrm{~km}^{-1}$, ie the absorption is 2 or 3 orders lower than copper wave-guides at microwave frequencies. Low dispersion in this band leads to a low "blurring" of the signal pulses and hence an enormous signal bandwidth. These considerations have been the driving forces to build photonic (also called lightwave) transmission systems, using electronic modulation of infra-red semiconductor lasers. The loss to convert from electrical to photonic signals and back gives a minimum distance over which photonic transmission gives lower net loss. The numerical value of this distance depends on data rates, signal-to-noise ratios and technological implementation, but typically it is $\approx 1 \mathrm{~km}$. Thus building 


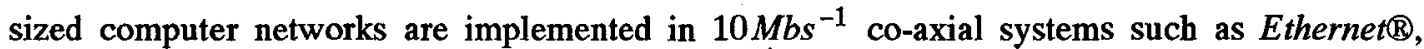
whereas campus sized networks may use $150 \mathrm{Mbs}^{-1}$ Fiber Distributed Data Interface (FDDI) ${ }^{\circledR}$ technology. This limit has restricted the use of photonics to $\mathrm{km}$ range transmission.

But recently it has been realized that photonics has another advantage, one which could broaden its use in the future. An infra-red photon at $0.8 \mathrm{eV}$ can generate an electron-hole pair in a $0.8 \mathrm{eV}$ band-gap semiconductor, which has only a few thermally generated carriers, from the room temperature thermal background, $k T=0.026 \mathrm{eV}$. On the other hand, an electronically generated $\mathrm{GHz}$ photon from a co-axial line has a photon energy in the $\approx \mu \mathrm{eV}$ range, and thus behaves classically, and requires many photons and much more energy to generate a detectable voltage in a classical detector ( $e g$ an antenna, tuned circuit and rectifier). This quantum behavior of infrared photon detectors can be used to effect what has been termed a "quantum impedance transformation" by Miller ${ }^{[1]}$ Briefly, he shows that information transmitted using photonic energies $(\approx 0.8 \mathrm{eV})$ can use less energy than that transmitted by low photon energy, classical $E M$ waves available from electronic devices. This fact takes on practical significance when it is realized that the performance (ie speed) of digital processors is ultimately limited by the energy dissipated per clock cycle: the processor must not generate more heat than its heat sink can remove at a safe processor operating temperature. A commonly used figure of merit is speedpower product, ie energy, which should be as low as possible for a given operation.

Thus the goal of digital processor designers is to use less energy per clock cycle, so that more cycles per second can be executed at constant power dissipation. (The parallel development of lower thermal impedance heat sinks is close to the ultimate limit set by nucleate boiling of refrigerant fluids such as Freon $\AA$. The cost of these is prohibitive in many cases.) Present electronic devices use $\approx 10^{6}$ more energy than the limit set by quantum thermodynamics, for switching. Nevertheless, most of the heat generated by an electron digital processor is in transmitting the signals from one chip of the processor to another: Every clock cycle, cables, of length $\approx l$, where $l$ is of order of the processor size, have to be fully charged and discharged, generating heat energy of $l C V^{2}$ where $C$ is the capacitance per unit length and $V$ is the voltage swing (typically $5 \mathrm{~V}$, but always $\gg 0.026 \mathrm{~V}$ ). Interestingly, this energy must be dissipated (in the load devices), even if the interconnections themselves are lossless (eg superconducting).

The quantum nature of infra-red photons enables photonic interconnection at much lower energy cost. The obstacle which prevents the use of this considerable advantage is a technological one related to the difficulty of manufacturing dense photonic circuits with a low switching energy. In terms of switching energy the very advantage that made photonic signals attractive in the first place, ie low interaction with matter, makes it rather difficult to devise a satisfactory photonic "transistor"; ie one with a high gain and a low switching energy. Such a device is necessary to obviate the need for wasteful conversion back and forth between electronic and photonic energy at each point in the processor.

One successful approach is the $S-S E E D$ device, a three terminal, all-photonic differential switch, whose operation relies on the quantum confined Stark effect ( $Q C S E)$, in which a roomtemperature exciton, confined in a thin quantum well structure, has its absorption peak shifted by application of a $D C$ bias, which can be optically controlled by a control beam. The shift in absorption peak modulates a power beam, to give the output beam. Efficient $Q C S E$ depends on a lack of absorption linewidth broadening since this controls the gain of the device. In present day $S-S E E D$ s, even at room temperature, the primary source of broadening is interfacial roughness.

This paper describes the efforts in hand to understand the nature of real quantum wells, whose properties are defined and determined by the interfaces between chemically different compounds, such as heterostructural $G a A s$ and $A l_{x} G a_{1-x} A s$. 


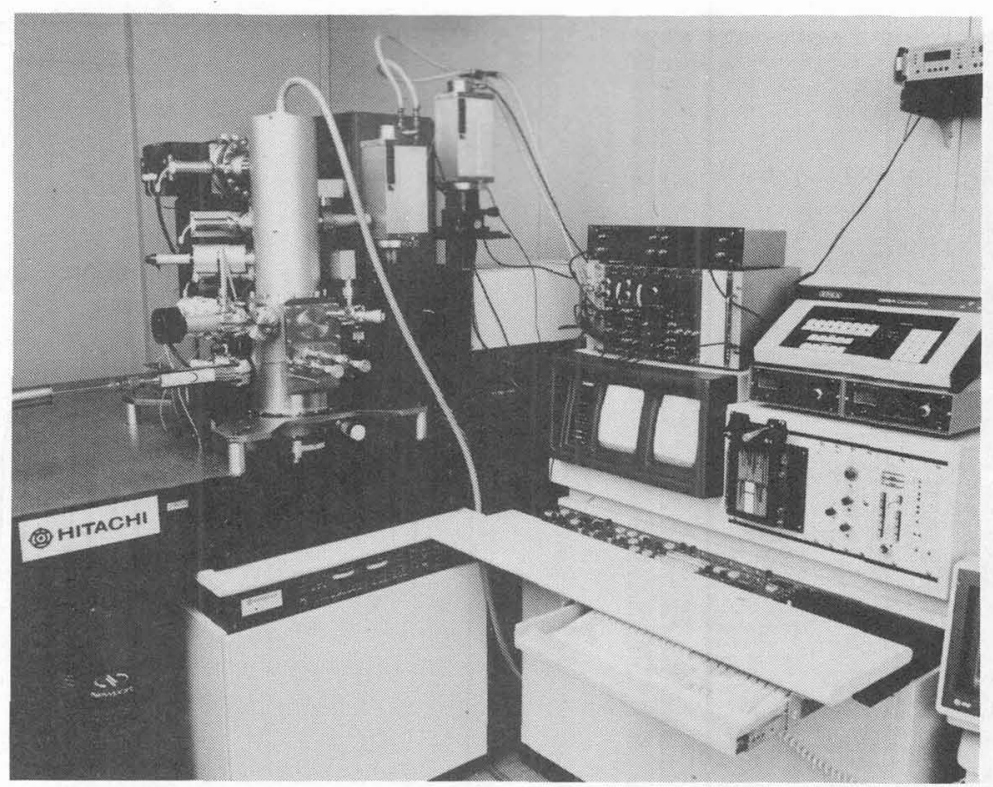

Figure 1. Scanning Cathodoluminescence Microscope in the author's laboratory

There are two difficulties in this work: the conceptual and practical difficulties of dealing with roughness on length scales which cover 8 orders of magnitude, and the fact that the interface is only defined between volumes of material sufficiently large to potentially contain significant numbers of $A l$ cations. The former problem is solved by considering the interface to be a $2-D$ waveform in Fourier space, having a amplitude vs spatial frequency spectrum spanning the huge range from $(\mathrm{cm})^{-1}$ to $(0.3 \mathrm{~nm})^{-1}$, and by compiling data from a variety of complimentary techniques, each covering a "window" of the frequency domain bounded in each case by a spatial resolution and a field of view. The latter problem is due to the fact that a $G a$ cation in $G a A s$ is indistinguishable from one in $A l_{x} G a_{1-x} A s$, and is considered by assigning a component of the roughness termed "random alloy roughness" to this effect.

\section{2.-Interfacial Roughness Studies}

\subsection{Transmission Electron Microscopy}

High quality interfaces are almost universally between compounds of the same structure but different chemical composition, with one exception, that of the $\mathrm{Si}_{/} \mathrm{SiO}_{2}$ interface.

Conventional diffraction contrast TEM is therefore unsuited to determining the interfacial structure. Chemical contrast in the HREM gives information on a length scale from near atomic dimension to 30 to $100 \mathrm{~nm}$, the field of view being restricted by the stringent sample preparation requirements. Ourmazd et $a l^{[2]}$ have demonstrated that the highest quality single quantum wells have interfacial roughness that can be characterized over the range of spatial frequencies mentioned above as having an Fourier amplitude of $0.3-0.6 \mathrm{~nm}$ and a peak spatial frequency of $\approx(2-3 n m)^{-1}$

\subsection{Photoluminescence}



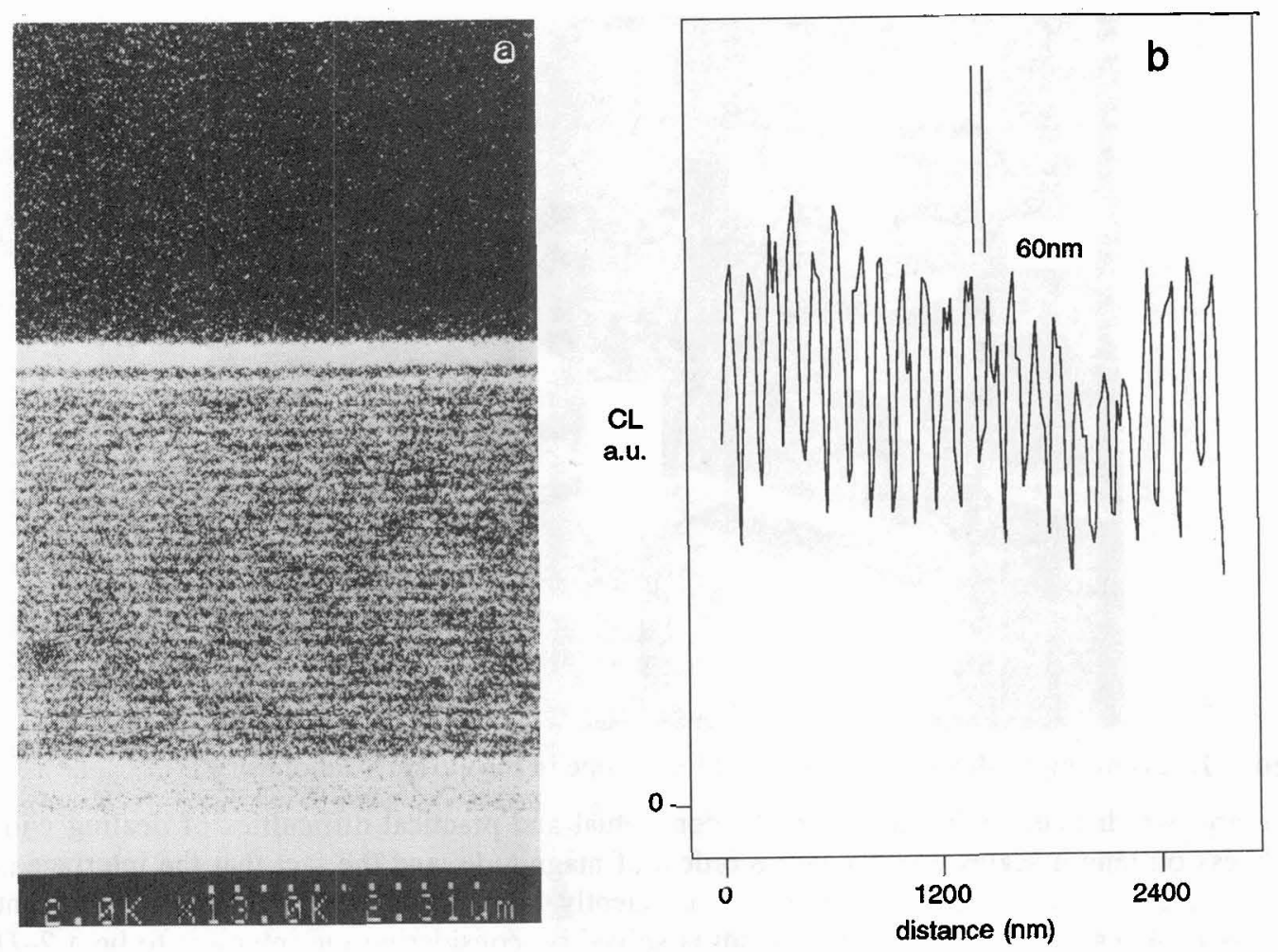

Figure 2. a) Cross-section of a dielectric stack mirror b) line trace from a). The interfaces are $60 \mathrm{~nm}$ apart

Weisbuch et al. ${ }^{[3]}$ were one of the first groups to use photoluminescence (PL) to infer an interface shape. However; as noted above it is difficult to form a complete picture of the interface by using only one technique. PL gives indirect information about how an ensemble of objects the size of the exciton $(\approx 15 \mathrm{~nm}$ diameter) experience a local average quantum well thickness. Goldstein et al. ${ }^{[4]}$ observed multiple peaks in PL spectra from single quantum wells, indicating a multivalued preferred thickness existed, and the difference in preferred thicknesses was close to one atomic layer or monolayer $(M L)$. [ $1 M L \approx 0.28 \mathrm{~nm}$ for $G a A s]$. This result gave indirect evidence to the idea that monolayer flat islands existed but when a more complete picture was formed from spatially resolved experiments, it became generally agreed that this picture was naive.

\subsection{Conventional Scanning Photoluminescence \& Cathodoluminescence}

Scanning luminescence can be conveniently achieved by using a focussed beam of photons or electrons (cathodoluminescence, $C L$ ). Bimberg et a ${ }^{[5]}$ have published detailed scanning $C L$ studies of high quality quantum wells. They emphasis the fractal nature of the interfaces, showing successively finer island-like features as the magnification is increased. Islands previously thought to be flat, proved to have sub-structure at bigher resolution, which shows they were not flat at all.

Further evidence that the mono-layer flat island picture was naive came from Warwick et $a l^{[6]}$ who showed the peak splitting varied by up to $38 \%$ for a given sample, which could not be 
accounted for by this picture. They proposed an interface shape which was characterized by a bimodal roughness spectrum.

PL and CL work motivated in part the desire for higher spatial resolution by several groups, including Wada et al. ${ }^{[7]}$ and the present author.

\subsection{Pre-diffusion Scanning Cathodoluminescence}

In the present work we have achieved $60 \mathrm{~nm}$ spatial resolution.

This is accomplished by:

a. using a low accelerating voltage to inject both near the surface and with minimal lateral scattering $(\approx 10 \mathrm{~nm})$

b. using a small probe generated by a bright, field-emission source

c. detecting photons from pre-diffusion radiative recombination events. Carriers living longer than $\approx 10 \mathrm{ps}$ recombine non-radiatively at the surface; the surface acts as a picosecond "shutter". We force surface recombination to dominate by injecting very close to the surface $(1 \mathrm{kV}$ electrons penetrate $\approx 10 \mathrm{~nm})$.
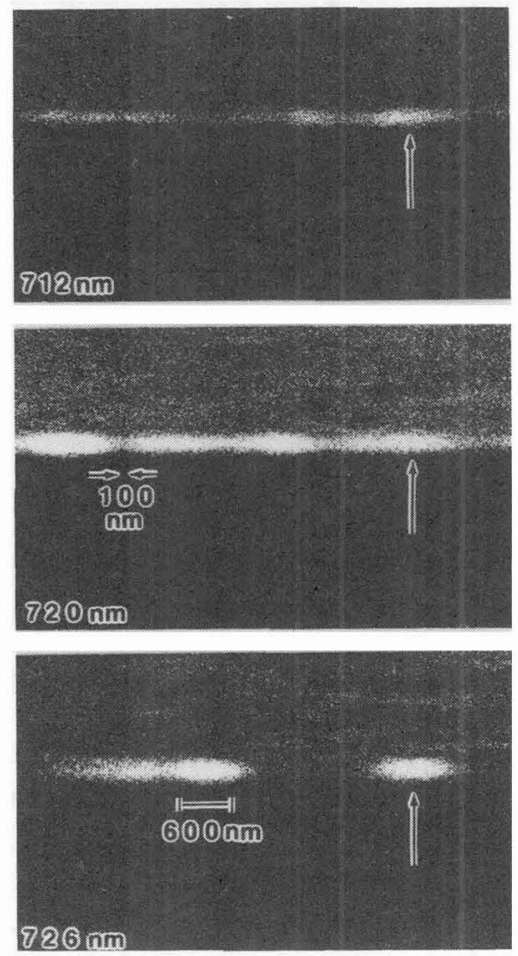

Figure 3. Cross-section of a single quantum well a) $712 \mathrm{~nm}$ CL b) $720 \mathrm{~nm}$ CL c) $726 \mathrm{~nm}$ CL

We also are aided in these efforts by efficient collection optics and a novel roof-mounted lowdrift $\left(\approx 0.02 \mathrm{~nm} \mathrm{~s}^{-1}\right)$ liquid helium sample holder (Oxford Instruments CF 302-SP). Sample surface temperature under excitation was determined to be $5 \mathrm{~K}$, from comparisons of PL and $\mathrm{CL}$ 
spectra from a high purity GaAs sample. Also vital are the Special Evacuation System on the specimen chamber of our Hitachi S-800-SES SEM which is oil-free and metal-sealed, and can withstand baking at $100^{\circ} \mathrm{C}$. As a result it has a base pressure (sample at room or helium temperature) below $5 \times 10^{-7} \mathrm{~Pa}\left(4 \times 10^{-8} \mathrm{Torr}\right)$. The low water vapor and hydrocarbon ambient practically eliminates the surface contamination that prevents low-temperature low-voltage observation in conventional SEMs.

In addition, the $S E M$ is mounted in a cut-out in an air-suspended optical table, thus eliminating vibration and aiding optical alignment. The detector is a thermo-electrically cooled GaAs photomultiplier tube used in photon counting mode. Counts are stored in direct digital form in a $4 M B$ frame buffer, memory-mapped into an $A T \& T 6386 E$ personal computer, which also controls the $0.75 m$ SPEX 1702 spectrometer. The frame buffer is part of an Hitachi Digital Image Processing System, that can also control the scan amplifiers. The microscope is shown in figure 1. Micrographs and spectra were recorded using $1 \approx 2 \mathrm{kV}$ electron beam with a probe current of $\approx 3 p A$.

\section{3.-Experimental Results}

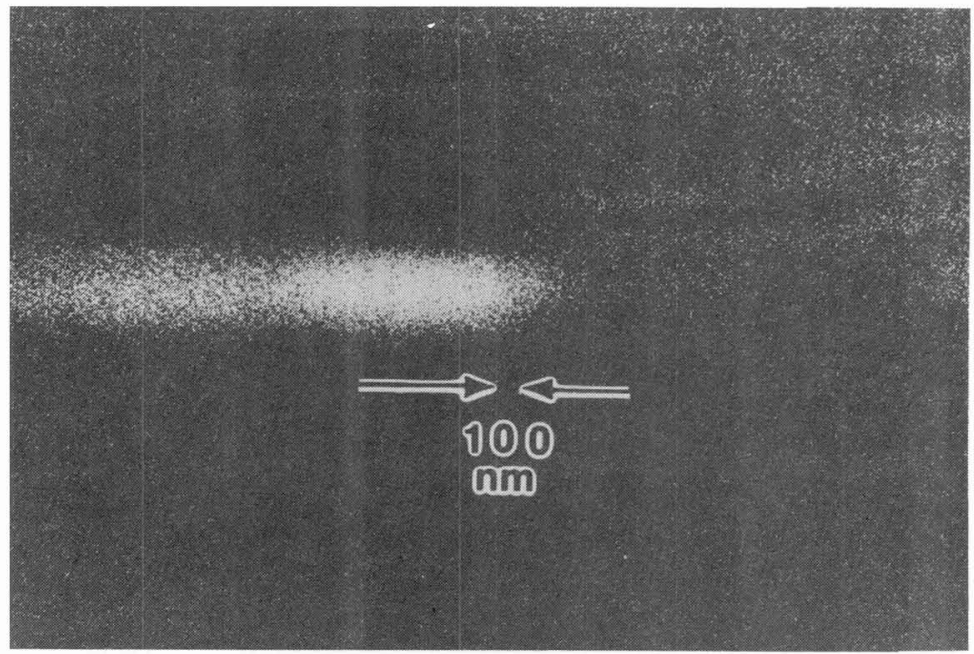

Figure 4. Cross-section of a single quantum well $726 \mathrm{~nm}$ CL: higher magnification

Figure 2a shows a cross-sectional Scanning Cathodoluminescence Micrograph of a dielectric stack mirror, used in $S-S E E D$ s and, as in this case, in Surface Emitting Lasers. The interfaces are $60 \mathrm{~nm}$ apart. Figure $2 \mathrm{~b}$ shows the line trace from this structure. The contrast is $\approx 50 \%$, easily satisfying the Rayleigh Criterion.

An important part of the $S-S E E D$ structure is its quantum wells. Figure 3 shows three monochromatic CL images of the same area of the same single quantum well, again viewed in cross-section. This type of quantum well is typical of one of the $\approx 30$ quantum wells used in $S-S E E D$ starting material. This sample has been studied previously using scanning PL and scanning tunneling microscopy by Kopf et al. ${ }^{[8]}$. In short, that paper showed that this sample has a remarkable macroscopic average thickness uniformity as evinced by the constant splitting of the 
PL peaks. However, the splitting observed corresponded to a change in quantum well width significantly less than $1 M L, \approx 0.9 M L$, which could be caused by fine scale roughness, superimposed on a low spatial frequency change in thickness. A detailed interpretation of the growth mechannism and resulting structure is in preparation ${ }^{[9]}$ but, referring to figure 3 , the most important thing to note is that the images at different wavelengths are partly complimentary and partly not. In other words, parts of the well emits light mainly at one of the three wavelength peaks but other sections emit at more than one wavelength, such as the region arrowed in figure 3. This is categorical proof that areas as small as $100 \mathrm{~nm}$ contain smaller areas of quantum well whose thicknesses are different by $\approx 1 M L$. The finer the resolution, the more detail is resolved. Thus the interfaces have a fractal like quality. Techniques with low resolution reveal only the low spatial frequency variation and give the misleading impression that large, monolayer-flat islands exist. Figure 4 shows the same sample at higher magnification, illustrating the high resolution that can be achieved by the method.

\section{4.-Conclusions}

High resolution Scanning Cathodoluminescence is giving us direct insight into the shape of interfaces which determine the properties of the quantum wells used in novel, experimental devices. As this work progresses and an understanding of the growth process is achieved, this will lead to better control and ultimately better devices.

\section{5.-Acknowledgments}

Rose Kopf and Benjamin Tell kindly provided the single quantum well sample and surface emitting laser sample respectively. Thanks are due to Abbas Ourmazd for many stimulating discussions.

\section{REFERENCES}

1. MILLER, D A B, Optics Letters 14 (1989) 146

2. OURMAZD, A, TAYLOR, D W, CUNNINGHAM, J, and TU, C W, Phys. Rev. Lett. 62 (1989) 933

3. WEISBUCH, C, DINGLE, R, GOSSARD A C, and WIEGMAN, W, Solid State Commun. 38 (1981) 709

4. GOLDSTEIN, L, HOROKOSHI, Y, TARUCHA S, and OKAMOTO $\mathrm{H}$, Japan. J. Appl. Phys. 22 (1983) 1489

5. BIMBERG, D, CHRISTEN, J, FUKUNAGA, T, NAKASHIMA, H, MARS, D E, and MILLER, J N, J. Vac. Sci. \& Technol. B5 (1987) 1191

6. WARWICK, C A, JAN, W Y, OURMAZD, A and HARRIS, T D, Appl. Phys. Lett 56 (1990) 2666

7. WADA, H, Japan J. Appl. Phys. Lett. 27 (1988) L1952

8. Kopf, R F, Schubert, E F, Harris, T D and BECKER, R S, Appl. Phys. Lett 58 (1991) 631

9. WARWICK, C A and KOPF, R F, Appl. Phys. Lett (1991) to be published 